\title{
Impact of Hospital Breast Feeding Awareness Among Lactating Mothers in Maternal and Children Hospital, AL-Hassa, Kingdom of Saudi Arabia
}

\author{
Esra O. Takrouni ${ }^{1}$, Rawabi A. Al Dawlah ${ }^{1}$, Shaimaa Khalifah ${ }^{1}$, Gihan Y. Ali ${ }^{1}$ \& Farah M. Al-Aithan ${ }^{1}$ \\ ${ }^{1}$ College of Medicine, King Faisal University, Al-Hassa, Kingdom of Saudi Arabia \\ Correspondence: Esra O. Takrouni, College of Medicine, King Faisal University, Al-Hassa, Kingdom of Saudi \\ Arabia. Tel: 966-554-827-720. E-mail: esratakrouni@gmail.com
}

Received: January 16, 2019 Accepted: February 24, 2019 Online Published: March 12, 2019

doi:10.5539/gjhs.v11n4p32 URL: https://doi.org/10.5539/gjhs.v11n4p32

\begin{abstract}
Background \& Aim: Insufficient knowledge and practice of breastfeeding may have serious disadvantages both on mother and child health. This study explores methods used in $\mathrm{MCH}$ based breastfeeding awareness program, level of benefit gained by newly delivered mothers after receiving the awareness, and the impact of mother's sociodemographic on the level of perceived benefit gained by them.
\end{abstract}

Subjects \& Methods: A prospective cross-sectional study applying random sampling technique was established. A self-administered questionnaire was distributed targeting newly delivered mother in maternal and child hospital in Al-Hassa, Saudi Arabia. It included two main parts: socio-demographic characteristics of the mothers, and questions related to the hospital breastfeeding awareness program.

Results: from the overall sample, hospital awareness was received by $47.5 \%$ of newly delivered mothers. The most common method to provide the knowledge was the Verbal demonstration representing 50\% of the mothers. They were followed by brochures representing 39\% and last, audios constituting only 3.6\%. From these methods, the verbal demonstration showed to be the one with the highest level of satisfaction and benefit reaching up to $85 \%$.

Conclusion: Breastfeeding awareness has a significant impact among both newly and non-newly delivered mothers with Hospitals playing a major role in this health education. The choice of method to provide breastfeeding awareness can contribute to the compliance of mothers as well as the level of their benefit and satisfaction.

Keywords: breastfeeding, hospital awareness, lactating mothers, newly mother

\section{Introduction}

Child care is essential for any mother. A number of studies demonstrated that mothers' education has a great influence on their practice concerning child health. One of the core aspects of the mother's education is the awareness regarding breastfeeding (Al-Ayed, 2010).

Breastfeeding is known to be an ancient and reliable method of infant feeding. Breast milk showed to enhance the physical, psychological and emotional Aspects of the infant as well as the breastfeeding mothers (Ogbeide et al., 2004). From an infant's health point of view, breastfeeding improves the nutritional status of infants as well as the immunological level by playing an essential role in preventing infections in the newborn (El Mouzan et al., 2009). From a maternal point of view, it is considered a natural instinct for any mother as it allows her to show her devotion and care for her children (Al-Amoud, 2003). A recommendation for breastfeeding has been issued by several professional agencies and societies including the American Academy of Pediatrics, the United Nations Children Funds (UNICEF) and the American Dietetic Association (Al-Amoud, 2003).

These recommendations described breastfeeding as the best way of feeding for newborn and infant. The World Health Organization (WHO) recommended exclusive breastfeeding for the first six months of life, giving the mother a choice to continue breastfeeding afterward (Senarath, Dibley \& Agho, 2010). This duration is vitally important to ensure a healthy life and wellbeing for those children. (Cwiek et al., 2010). From a health care point of 
view, breastfeeding is seen as a public health strategy for improving infant, child, and maternal mortality and morbidity. It also helps to control health care costs and expenditure (Al-Binali, 2012).

As an integrated part of maternal and child health, health care professionals are obligated to provide updated education for mothers regarding the benefit of breastfeeding (Alwelaie et al., 2010). All medical staff and midwives should offer information as well as emotional and technical support for mothers throughout the entire period of lactation (Klejewski et al., 2012).

\subsection{Research Questions}

- What are the methods used for awareness in hospital-based breastfeeding awareness program?

- How did mothers perceive these methods as being beneficial?

- What is the relation between mother's sociodemographic and the level of perceived benefit gained by them?

\subsection{Aim}

To explore methods used in Maternal and Children Hospital based breastfeeding awareness program, level of benefit gained by newly delivered mothers after receiving the awareness, and the impact of mother's sociodemographic on the level of perceived benefit gained by them.

\section{Literature review}

Several previous studies about improving breastfeeding knowledge, attitudes, and practices have documented the importance of health professional support for breastfeeding promotion (Khoury et al., 2002).

These studies indicate the vital role of hospital-based breastfeeding program to improve the overall knowledge and practice of mothers.

In Saudi Arabia, the awareness done in hospitals regarding the importance of breastfeeding have shown low levels. Several studies were done to investigate breastfeeding practice and factors affecting it in the kingdom of Saudi Arabia (Alwelaie et al., 2010), (Al-Hreashy et al., 2008). Most of these studies were done in Riyadh, and others were done in al-Khobar, Abha and Al- kharj regions.

\subsection{First, Four Studies Were Done in Riyadh}

In 2009 a study was conducted in three major hospitals in Riyadh to examine Saudi mother's knowledge and practice regarding breastfeeding. The study included eight hundred and forty-eight mothers during their post-delivery period as well as mothers attending the antenatal clinics. An Arabic questionnaire was distributed and answered by the targeted population. The results showed that only $55.8 \%$ have a prior education regarding breastfeeding, and about $54.2 \%$ received it during their stay in the hospital. About $48.5 \%$ used mixed feeding, on the other hand (36.8\%) preferred exclusive breastfeeding during the first six months of life. Different reasons were mentioned, for example, illness of the baby (38.2\%), secession of breastfeeding for medical reasons $(61.1 \%)$, mother sickness or consumption of certain medication (71.8\%), at last, the use of contraception (32.8\%). The study concluded that practice of exclusive breastfeeding was not conducted according to recommendation raising the need for more targeted awareness program especially from the health care society as half of the women receives their first education in the hospital (Alwelaie et al., 2010).

The second study was carried out in the same city including four primary care centers, and well-baby clinic at King Abdulaziz Medical City. It investigated a total number of 578 infants ranging from 6 to 7 months old. An Arabic questionnaire was generated and distributed gathering data related to mothers and their children and breastfeeding habits. The result showed that initiation of breastfeeding reached up to $95 \%$ despite the length of breastfeeding. However, the majority of mothers $(83.7 \%)$ indicated to give milk formula during the first six months with $(75.7 \%)$ introducing it at the neonatal period. It was noticed that most of the infants $(94.3 \%)$ had an addition to their diet during the same period in the form of water, baby tea, herbs, and dates. About (89.2\%) introduced baby food to their infants approximately by the age of 4-6 months. The study demonstrated as well an inverse relationship between the formula introduction and the lactation duration. It also showed a decline by half in numbers of lactating mothers in comparison to initiation at the age of 6 months. The most common reasons included insufficient milk (49.6\%), and breastfeeding difficulties (11.6\%), which were gathered from responses like: "refusal of the breast," "improper latch on," "sore nipple," "pain" and "infection (Al-Hreashy et al., 2008).

The next study was done at the pediatric outpatient clinic of King Khalid University Hospital. The sample included 373 questionnaires distributed among mothers attending the clinic. The survey included two parts. The first part contained information about the mother's sociodemographic data, and the second part comprises questions 
regarding child well-being and health. A total score of 40 was given for the statements in the questionnaires with the highest score of 40 , and the reported score of 25 declared as satisfactory. Results of the study showed that The mean knowledge score was 25 (out of 40), the least score recorded was 14, and the highest was $36.58 .4 \%$ of mothers scored 25 or more. The primary source of health information was $(80 \%)$ family members, $(27.1 \%)$ books, $(8.2 \%)$ schools, (17.2\%) TV and radio, (16\%) journals and magazines, and only (7.1\%) for health care professionals. It concluded that mothers knowledge in child health is considered deficient, as well as the Health care sector having an insufficient contribution to maternal awareness. Therefore, more effort should be generated to improve the mother's education regarding child health. This matter should be addressed by school and health care facilities as well (Al-Ayed, 2010).

The last study done in Riyadh was nationwide nutritional survey included 5339 mothers of children less than three years of age as part of the Health Profile for Saudi Children

and Adolescents Project. The dietary section of a validated questionnaire was administered. It included items regarding the initiation and timing of breastfeeding as well as barrier to breastfeeding continuation. The result showed an attitude of initiation of breastfeeding in $91 \%$ of the mothers. It was followed by a drop in the attitude of breastfeeding from $88.6 \%$ at birth to $1.8 \%$ at 12 months. Nearly $80 \%$ of the infants were started on bottle milk formula by four and six months, respectively. "Solid" food was given to $81.5 \%$ of infants by the age of 4 to 6 months. The study demonstrated that many mothers are willing to initiate breastfeeding, but many fail to continues until the period recommended by the WHO. Awareness regarding breastfeeding should be made a public health priority, and more research should be done regarding this aspect of child health (El Mouzan et al., 2009).

\subsection{Second, one study was conducted in Al-Khobar city, Eastern province.}

It analyzed the predictor factors affecting exclusive breastfeeding practice. Around 400 fertile women with living children, less than five years of age agreed to take part in the study. Data were collected using an interview questionnaire which was composed of two main elements: socio-demographic characteristics, and questions assessing the level of mother's knowledge. The result demonstrated the total percent of mothers with adequate knowledge and practice toward breastfeeding were $91.8 \%$, and $91.5 \%$, respectively. $79.5 \%$, used mixed feeding (breastfeeding complemented by bottle feeding). Only 19.2\% of the studied women used exclusive breastfeeding. Family size and paternal education were the most influential factors that influenced the continuation of exclusive breastfeeding. The study concluded that Knowledge and practice of Saudi women regarding breastfeeding were satisfactory, but their practice of absolute breastfeeding was less than adequate. Therefore, promoting and protecting the practice of exclusive breastfeeding need to be adequate (Al-Yousif et al., 2011).

\subsection{The third study was done in Abha Southwestern region, Saudi Arabia}

The total number of participants were 384 women who met the criteria of being school teachers having a child aged five years or less. The questionnaire was structured using the Delphi technique and was distributed among the targeted population. It included two sections, first: demographic, and biological data, second: questions examining knowledge and attitude toward exclusive breastfeeding. The study showed that $31 \%$ of mothers had breastfed their infants for the first time directly after delivery. $90.9 \%$ of the respondent had mixed feeding in the form of breast milk combined with formula feeding. While only $9.1 \%$ breastfed their children exclusively. $8.3 \%$ continue to breastfeed solely till the period of 6 months while others $44 \%$ contributed the discontinue of breastfeeding to reasons such as Insufficient breast milk and work burden. As for mother's willingness for education regarding breastfeeding, $8.6 \%$ had already participated in classes related to breastfeeding education, but the majority representing $68 \%$ showed interest to participate in such courses, if available in future pregnancies (Al-Binali, 2012).

2.4 Another study was carried out in Al kharj city discussing the influencing factors affecting exclusive breastfeeding as well

In this study, 704 questionnaires were distributed among women in childbearing age visiting Al Kharj Health Centre. Participants were grouped according to their status regarding breastfeeding into exclusive feeding, mixed feeding (breastfeeding with bottle feeding), and bottle feeding. Results showed that mixed feeding was the dominant mode of infant feeding representing $66.1 \%$ of mothers. Exclusive breastfeeding was the next most common representing $27.3 \%$. Finally, exclusive bottle feeding showed to be the lowest representing $6.7 \%$ of mothers. Four main factors proved to be significant regarding the exclusive mode of breastfeeding; it included partner's educational level, non professional breastfeeding advices, bottle feeding being given among discharge, and the use of contraception (Ogbeide et al., 2004).

Both studies concluded that mixed feeding or partial breastfeeding is the dominant practice for the majority of 
mothers. It also illustrated the importance of breastfeeding awareness as most mothers are willing to practice breastfeeding if they had an appropriate awareness method.

\subsection{Finally, the last study was done in Al-HASA, Saudi Arabia}

The study analyzed the Determinants of Initiation and Exclusivity of Breastfeeding in Al Hassa region. A sample of 641 mothers with infants aged 24 months or less was interviewed. The results demonstrated that $77.8 \%$ of mothers were willing to breastfeed within the first 24 hours. This rate showed a progressive decline during the first six months reaching up to $12.2 \%$. Several factors showed to affect the practice of breastfeeding such as mothers age, education, and work status. The study emphasized the presence of various misconception regarding breastfeeding among mothers. About $47.0 \%$ thought that breastfeeding causes increase in weight and change the breast shape. Around $60.0 \%$ believed that pregnancy is a contraindication for breastfeeding. $28.9 \%$ agreed to stop breastfeeding if stomachache occurred like in the cases of diarrhea. $32.3 \%$ confirmed that mixed feeding should be introduced by three months of age, and $55.9 \%$ failed to define the recommended duration for exclusive breastfeeding. The study concluded that the mother's level of education and awareness is far behind the WHO goal. More comprehensive and long term awareness programs are needed to help mothers reach the desired benefit indicated by the WHO (Amin, Hablas \& Al Qader, 2011).

\section{Subject and methods}

\subsection{Study design and data collection}

A random prospective cross-sectional study was conducted on maternal and children hospital in Al-Hassa, Saudi Arabia, in the year of 2014 for one month.

The study sample included Saudi women who newly delivered their baby, excluding non-Saudi mothers or those who exceeded the current hospital admission. One hundred mothers were interviewed, 61 out of them met the research criteria.

After obtaining the consent, Well-trained medical students administered a questionnaire using a face to face interview; the primary goal was to examine the impact of hospital breastfeeding awareness on their current practices.

\subsection{Research tool}

An Arabic questionnaire was distributed among the mothers.

The questionnaire was designed based on data collected from previously conducted studies testing the factors impacting breastfeeding awareness among mothers. The survey was tested to suit the availability of awareness in MCH hospital. Finally, it was reviewed and approved by a valid professor and analyst. After which it was distributed among the participating mothers.

It included Socio demographical data: the age of mothers in years, educational status, number of children, previous knowledge about breastfeeding, the source of this knowledge and whether those mothers had hospital awareness or not. According to their state regarding hospital awareness, the studied mothers were grouped into two groups. Group I (mothers who received hospital awareness, and Group II (mothers who did not receive hospital awareness).

\subsection{Data management and analysis}

The data were assembled and analyzed using SPSS version 17. Nominal variables were expressed using mean, SD and range. Categorical variables were expressed using numbers and percentage.

A chi-square test was applied to define the relationships between the different categorical variables. The significance was determined to be less than 0.05 .

\subsection{Ethical considerations}

Approval was obtained from the college's research committee, and the permission \& acceptance of the ethical and research committee of the MCH hospital and Ministry of Health was taken as well.

Written approval was acquired from all the participants in this study after they were informed about the aim, and content of the study. Participants were reassured about the full confidentiality of their data. All the mothers agreed to participate in the questionnaire.

\section{Results}

This study was conducted among 61 lactating mothers who were interviewed by medical students from KFU colleague of medicine and had a $100 \%$ response rate. The socio-demographic characteristics of the mothers, the 
number of mothers who received and did not receive the awareness from the hospital are shown in Table 1.

Table 1. Distribution of studied women according to their socio-demographic characteristics in the selected hospital $(\mathrm{n}=61)$

\begin{tabular}{lll}
\hline & $\begin{array}{l}\text { Group I(RHA)* } \\
\mathbf{2 9}(\mathbf{4 7 . 5 \% )}\end{array}$ & $\begin{array}{c}\text { Group II(NRHA)* } \\
\mathbf{3 2 ( 5 2 . 5 \% )}\end{array}$ \\
\hline Age (mean \pm SD) & $30 \pm 5.9$ & $27.7 \pm 6.4$ \\
\hline Number of Children & $9(31 \%)$ & $12(37.5 \%)$ \\
$\mathbf{0}<\mathbf{1}$ & $12(41 \%)$ & $15(46.8 \%)$ \\
$\mathbf{1 - 3}$ & $8(27.5 \%)$ & $6(18.7 \%)$ \\
$>\mathbf{3}$ & & \\
\hline Previous knowledge & $27(93 \%)$ & $22(68.7 \%)$ \\
Knowledgeable & $2(6.9 \%)$ & $10(31 \%)$ \\
Non-knowledgeable & & $10(31 \%)$ \\
\hline Effect of Previous knowledge & $15(51.7 \%)$ & $12(37.5 \%)$ \\
Effective & $12(41 \%)$ & \\
Non- effective & & $2(6.2 \%)$ \\
\hline Education status & $1(3.4 \%)$ & $5(15.6 \%)$ \\
Illiterate & $1(3.4 \%)$ & $1(3 \%)$ \\
Elementary & $5(3.4 \%)$ & $5(15.6 \%)$ \\
Intermediate & $14(48 \%)$ & $13(40.6 \%)$ \\
High school & $6(20.6 \%)$ & $2(6 \%)$ \\
Bachelor & - & \\
Seminar & &
\end{tabular}

*Note. $\mathrm{RHA}=$ received hospital awareness, NRHA=did not Receive hospital awareness.

As demonstrated in Table 1, the number of mothers in group I (mothers who received hospital breastfeeding awareness) were less than group II (mothers who did not receive hospital breastfeeding awareness) with a rate of (47.5\%) in comparison to (52.5\%). The mean age of mothers in group I was 30.1 parallel to 27.7 for those in group II. Most of the mothers in group I (41.3\%) had from one to three children in relation to (46.8\%) in Group II. About $93 \%$ of group I had previous knowledge about the importance of breastfeeding. Most of those mothers $51.7 \%$ acknowledged gaining benefit from their previous knowledge. In group II $68.7 \%$ of mothers had prior knowledge about breastfeeding, among them $31.2 \%$ acknowledged to gain benefit as well. Sociodemographic characteristics showed that the majority of mothers in group I had a high school education representing $48 \%$ and bachelor degree representing $20.6 \%$.

Table 2. Mother's perceived benefit from variable awareness methods.

\begin{tabular}{llllll}
\hline & \multicolumn{2}{l}{ Total number of mothers receiving hospital awareness $\mathbf{2 9} \mathbf{( 4 7 . 5 \% )}$} \\
\cline { 2 - 6 } & Very good & Good & Nothing & Total & Significance \\
\hline Brochure & $9(81.8 \%)$ & $2(18.2 \%)$ & - & $11(39 \%)$ & 0.6 \\
\hline Verbal & $12(85.7 \%)$ & $1(7 \%)$ & $1(7 \%)$ & $14(50 \%)$ & 0.4 \\
\hline Audio & $1(3.6 \%)$ & - & - & $1(3.6 \%)$ & 0.9 \\
\hline Brochure + Orally & $3(10.7 \%)$ & - & - & $3(10.7 \%)$ & 0.8 \\
\hline
\end{tabular}


As noted in Table 2, most of the mothers in group I who were involved in the hospital breastfeeding awareness program perceived to gain more benefit from Verbal education in comparison to other methods of awareness. 50\% of the mothers received the knowledge in the form of Verbal training delivered by the hospital staff and health care educator. It was Followed by $39 \%$ who received brochures with all the information, but no demonstration was done. Education of $3.6 \%$ of mothers was performed through audio records, and $10.7 \%$ received both brochure and verbal awareness. Based on data analysis, verbal instruction showed a significant of 0.4 in comparison to other methods of awareness. It demonstrated no significant relationship between the modes of awareness and the level of mother's benefit. But referring to the given clinical significant which showed the majority $85 \%$ demonstrated to have greater benefit from oral education as a method for awareness.

Table 3. Association between benefit from hospital awareness and mother's educational status

\begin{tabular}{llll}
\hline & \multicolumn{2}{l}{ Benefit from hospital awareness } & Total \\
\cline { 2 - 4 } & Yes & No & $6(20.6 \%)$ \\
\hline High Education & $6(100 \%)$ & - & $21(72.4 \%)$ \\
Low Education & $20(95.2 \%)$ & $1(4.7 \%)$ & \\
\hline
\end{tabular}

As shown in Table 3, it was observed that $20.6 \%$ of the mothers representing highly educated mothers (having high school education and above) had $100 \%$ benefit from the awareness in contrast $72.4 \%$ serving low educated mothers with $95.2 \%$ of them perceived to have profited from the awareness program. It was noticed that most of those mothers got to benefit from the awareness as it either added to their previous knowledge or corrected false information. When analyzing the data, it was determined that there is no statistical significance between the educational status and the level of benefit for breastfeeding.

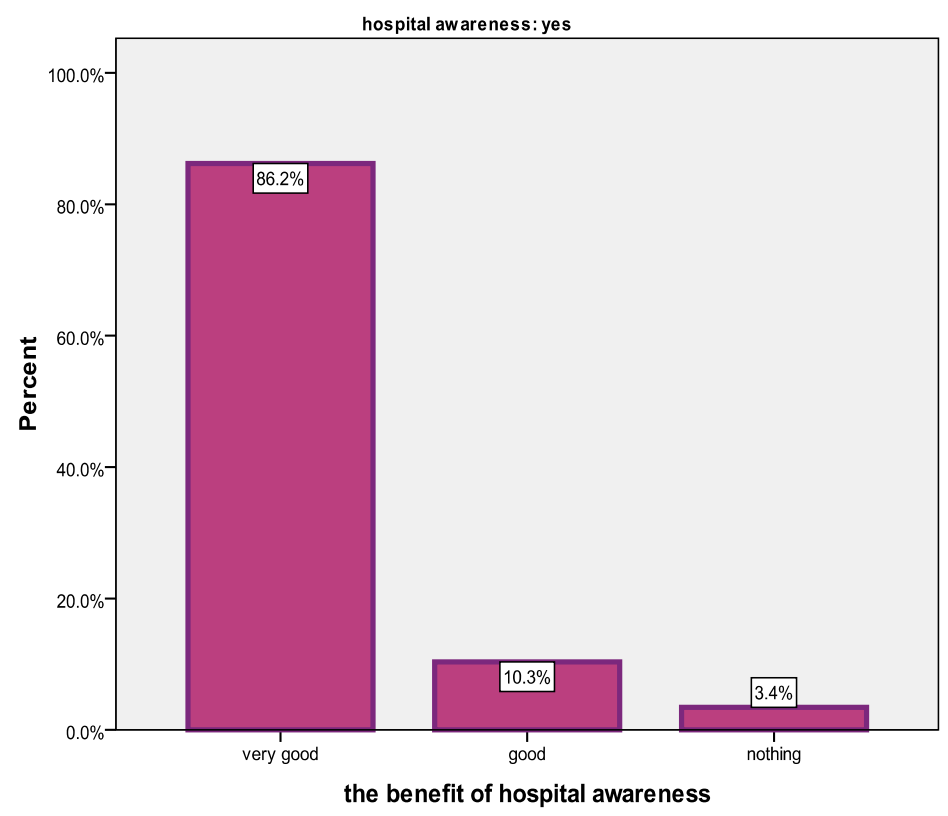

Figure 1. Distribution of studied mothers according to their benefits from hospital awareness

Depending upon illustration shown in Figure 1, the hospital awareness program demonstrated to be effective comparing the evaluation of levels of benefit given by the mothers. $86.2 \%$ from the mothers who received the hospital awareness evaluated the benefit as very good, followed by $10.3 \%$ who evaluated the benefit as good knowledge, the remaining 3.4\% didn't perceive to gain any benefit from the hospital awareness.

The questionnaire interview viewed the option of having an educational seminar regarding breastfeeding awareness. Around $62.9 \%$ of the mothers who did not obtain hospital awareness showed enthusiasm to attend as they perceive it as a reliable source of knowledge. 


\section{Discussion}

Worldwide feeding limited to mother's milk is the optimal method for child development and well-being. Countless benefits for both maternal and child health are well proved in variable literature (Al-Yousif et al., 2011). Our study aimed to explore methods used in $\mathrm{MCH}$ based breastfeeding awareness program, level of benefit gained by newly delivered mothers after receiving the awareness, and the impact of mother's sociodemographic on the level of benefit gained by them.

Considering this purpose, the prevalence of mothers receiving hospital awareness was only $47.5 \%$. Our study has shown that maternal age has a significant impact on the level of benefit perceived by mothers from breastfeeding awareness program. It demonstrated that older mothers perceive more benefit from awareness in comparison to the younger one. This finding was compatible with the previous study done by (Al-Yousif et al., 2011) from Saudi Arabia, which has reported that maternal age was a significant independent predictor for breastfeeding initiation and feeding choice (Al-Yousif et al., 2011). Another study was done by Al-Jassir et al., (2006), disagreed with the proposed result as they concluded that the younger generations were more informed and enthusiastic than older mothers (Al-Jassir et al., 2018). Concerning the other factor which is parity, our study concluded that there is no significant influence of the parity of mothers in their attitude toward breastfeeding initiation and duration. This conclusion was opposite to what was demonstrated by Trussel et al., (2011); concluded that the higher parity mothers have the longest they tend to breastfeed their infants (Trussell et al., 1992). In this study, the relationship between different methods used for awareness and the level of mother's knowledge was described as being satisfactory. Similar results were obtained from a questionnaire conducted by Al-Yousif G et al. (2011); which demonstrated a satisfactory knowledge and attitude for Saudi mothers toward breastfeeding (Al-Yousif et al., 2011). However, the current study demonstrated the effectiveness of awareness method in relation to the level of mothers' benefit, unlike the previous studies where the overall knowledge was evaluated regardless of the reference or awareness program role in obtaining this knowledge.

In our study, we found that there is no statistically significant effect of educational status in the level of benefit obtained from the awareness program, but the considerable results show high education level are more likely to gain benefit from the awareness giving a percentage of $100 \%$ in comparison to low education status having a rate of 95.2\%. This finding was compatible with other studies done by Al-Yousif G et al., (2011), and Amin, (2011). The first study indicated no statistically significant difference in the level of knowledge of studied women as $91.8 \%$ of all studied women had overall good knowledge. The second study demonstrated no statistically significant effect, but the mean knowledge score indicated that secondary education or higher score better regarding the knowledge part, 10.21 out of 14 vs. 8.4 out of 14 for less than secondary education (Al-Yousif et al., 2011; Amin, Hablas \& Al Qader, 2011).

Furthermore, this study described the distribution of mothers according to their evaluation of the hospital awareness program. Around $86 \%$ representing the majority of the studied mothers indicated that the awareness program positively influenced their level of knowledge as well as their attitude, and intention to continue breastfeeding. Encouraging them to initiate breastfeeding as well as counseling them regarding the proper steps for feeding were the main items influencing the higher level of satisfaction. Recent reviews were done by Meedya $\mathrm{S}$ et al. (2010); and Hong Jiang et al. (2012); argued that breastfeeding intention was a good predictor for breastfeeding initiation and duration (Meedya, Fahy \& Kable, 2010; Jiang et al., 2012).

\section{Conclusion}

The current study concluded that Hospital breastfeeding awareness program could represent a cornerstone for maternal and child health education and awareness. As breastfeeding habits diminish with the availabilities of alternatives, modernized and effective methods must be integrated into these programs and regularly followed up. Helping mothers to have a pleasant breastfeeding experience through the journey of motherhood can improve their compliance and thus the ability to reintegrate this habit into society again.

\subsection{Limitation}

This study is not without limitations, first of all, the studied population included a small sample from a single hospital integrated with the awareness program. This limited evaluation leaves a vast space for further studies to be implemented as this field of research remains to be lacking. Further studies may include multihospital assessment of breastfeeding awareness program. Several cities to be compared as social norm limiting breastfeeding vary from one region to the other. Second, follow up studies should be done taking into consideration the technological revolution allowing innovative methods for awareness to be implemented. Those methods can be integrated to enhance the quality of these awareness programs. 


\subsection{Recommendation}

1. Breastfeeding awareness programs should be more integrated into society and not limited to the mother's hospital stay. Establishing breastfeeding clinics as part of the hospital awareness programs can improve maternal compliance as most of the mothers' address variable difficulties in breastfeeding after being discharged from the hospital.

2. More updated and technology friendly media could be used to give mothers education and counseling. Furthermore, the continuity to them support them and follow them outside the hospital boundaries is becoming a necessity.

3. Interactive classes and campaigns could be established as part of a breastfeeding awareness program to help raise the awareness in the society as well as becoming the initial step to help more mothers getting involved into the services of those awareness programs.

\section{Acknowledgments}

We want to use this opportunity to thank our supervisor, Dr. Gihan Yousef Ali for providing us with supervision, guidance, and support until the completion of our research. Her effort and teaching was the founding stone for this research.

We also like to express our appreciation for those who agreed thankfully to participate in our research.

We would like to deliver our sincere appreciation for KING FAISAL UNIVERSITY colleague of medicine for giving us support and professional guidance.

In addition, we would like to thank Maternal and Children Hospital in Al-Hassa for being a motivated, and welcoming institution for our research to be conducted in the ground of their hospital.

Last but not least, our appreciation will be incomplete without a special reference to our families, friends, and colleagues who were the greater support and encouragement of all

Finally, we dedicate this research to our parents who were the opened arms in all the difficult time we faced until the conclusion of this research.

\section{Competing Interests Statement}

The authors declare that there are no competing or potential conflicts of interest.

\section{References}

Al-Amoud, M. (2003). Breastfeeding Practice Among Women Attending Primary Health Centers in Riyadh. Family Community Med, 10(1), 19-30.

Al-Ayed, I. (2010). Mothers' Knowledge of Child Health Matters: Are We Doing Enough? Journal of Family and Community Medicine, 17(1), 22. https://doi.org/10.4103/1319-1683.68785

Al-Binali, A. (2012). Breastfeeding Knowledge, Attitude and Practice Among School Teachers in Abha Female Educational District, Southwestern Saudi Arabia. International Breastfeeding Journal, 7(1), 10. https://doi.org/10.1186/1746-4358-7-10

Al-Hreashy, F., Tamim, H., Al-Baz, N., Al-Kharji, N., Al-Amer, A., Al-Ajmi, H., \& Eldemerdash, A. (2008). Patterns of Breastfeeding Practice During the First 6 Months of Life in Saudi Arabia. Kingdom of Saudi Arabia. Saudi Med J, 29(3), 427-31.

Al-Jassir, M., El-Bashir, B., Moizuddin, S., \& Abu-Nayan, A. (2018). Infant Feeding in Saudi Arabia: Mothers' Attitudes and Practices. Riyadh, Saudi Arabia. East Mediterr Health J, 12(2), 6-13.

Al-welaie, Y., Alsuhaibani, E., Al-Harthy, A., Radwan, R., Al-Mohammady, R., \& Almutairi, A. (2010). Breastfeeding Knowledge and Attitude Among Saudi Women in Central Saudi Arabia. Saudi Med J, 31(2), 193-198.

Al-Yousif, G., Sabra, A., Sebiany, M., \& Hafez, S. (2011). Predictors of Breastfeeding Practices in Primary Health Care Facilities at Al-Khobar City, Eastern Saudi Arabia. The Egyptian Journal of Community Medicine, 29(3), pp.13-24.

Amin, T., Hablas, H., \& Al Qader, A. (2011). Determinants of Initiation and Exclusivity of Breastfeeding in Al Hassa, Saudi Arabia. Breastfeeding Medicine, 6(2), 59-68. https://doi.org/10.1089/bfm.2010.0018

Cwiek, D., Branecka-Woźniak, D., Fryc, D., Grochans, E., \& Malinowski, W. (2010). Reasons for Giving Up Breastfeeding and Support During Problems with Lactation in The North-Western Part of Poland. Ann Acad 
Med Stetin, 56(2), 129-32.

El Mouzan, M., Al Omar, A., Al Salloum, A., Al Herbish, A., \& Qurachi, M. (2009). Trends in Infant Nutrition in Saudi Arabia: Compliance with WHO Recommendations. Annals of Saudi Medicine, 29(1), 20. https://doi.org/10.4103/0256-4947.51812

Jiang, H., Li, M., Yang, D., Wen, L., Hunter, C., He, G., \& Qian, X. (2012). Awareness, Intention, And Needs Regarding Breastfeeding: Findings from First-Time Mothers in Shanghai, China. Breastfeeding Medicine, 7(6), 526-534. https://doi.org/10.1089/bfm.2011.0124

Khoury, A., Hinton, A., Mitra, A., Carothers, C., \& Foretich, C. (2002). Improving Breastfeeding Knowledge, Attitudes, And Practices of WIC Clinic Staff. Public Health Reports, 117(5), 453-462. https://doi.org/10.1016/S0033-3549(04)50186-3

Klejewski, A., Urbaniak, T., Baczyk, G., \& Cichocka, E. (2012). Knowledge about Breast Feeding Advantages among Primiparas. PrzeglLek, 69(10), 1021-5.

Meedya, S., Fahy, K., \& Kable, A. (2010). Factors That Positively Influence Breastfeeding Duration to 6 Months: A Literature Review. Women and Birth, 23(4), 135-145. https://doi.org/10.1016/j.wombi.2010.02.002

Ogbeide, D., Siddiqui, S., Al Khalifa, I., \& Karim, A. (2004). Breast Feeding in A Saudi Arabian Community. Profile of Parents and Influencing Factors. Saudi Med J, 25(5), 580-4.

Senarath, U., Dibley, M., \& Agho, K. (2010). Factors Associated with Nonexclusive Breastfeeding in 5 East and Southeast Asian Countries: A Multilevel Analysis. Journal of Human Lactation, 26(3), 248-257. https://doi.org/10.1177/0890334409357562

Trussell, J., Grummer-Strawn, L., Rodriguez, G., \& Vanlandingham, M. (1992). Trends and Differentials in Breastfeeding Behaviour: Evidence from The WFS and DHS. Population Studies, 46(2), 285-307. https://doi.org/10.1080/0032472031000146246

\section{Copyrights}

Copyright for this article is retained by the author(s), with first publication rights granted to the journal.

This is an open-access article distributed under the terms and conditions of the Creative Commons Attribution license (http://creativecommons.org/licenses/by/4.0/). 